\title{
Endometriosis/adenomyosis is associated with more typical cystoscopic findings of interstitial cystitis in patients with elevated PUF scores
}

\author{
Richard L. Heaton • M. Sami Walid
}

Received: 17 March 2010/Accepted: 23 April 2010 /Published online: 12 May 2010

(C) Springer-Verlag 2010

\begin{abstract}
In this paper, we present our experience with cystoscopy and hydrodistension for the diagnosis of interstitial cystitis (IC) and association with endometriosis/ adenomyosis. This is a retrospective study of 116 patients who have undergone cystoscopy and hydrodistension because of suspicion of interstitial cystitis as shown by elevated Pain Urgency and Frequency questionnaire (PUF) scores and, in some patients, positive potassium sensitivity test (PST) or anesthetic bladder challenge (ABC) tests as well. Cystoscopic findings were grouped into "no IC," "atypical IC," and "typical IC" groups. Chi-square analysis was used for comparing percentages. PUF questionnaire showed high sensitivity (98\%) with $\geq 5$ score but low specificity $(0 \%)$. The best specificity was found with PST (30\%). Among IC patients, $60(60 \%)$ had adenomyosis or endometriosis with an increasing endometriosis/adenomyosis rate from "no IC" to "typical IC" groups $(37.5 \%$ to $67.74 \%, P<0.05)$. PST is the most specific of the screening tests for IC, and cystoscopy with hydrodistension is the only test that allows direct visualization and grading of bladder abnormalities as "typical", "atypical", and "no IC".
\end{abstract}

Declaration of interest Dr Heaton is a consultant for Ethicon Women's Health \& Urology and a speaker for Ortho Women's Health \& Urology. Dr Walid has no affiliations that may pose a conflict of interest. No funding was received for preparing this paper.

\footnotetext{
R. L. Heaton $(\bowtie)$

Heart of Georgia Women's Center, 209 Green Street,

Warner Robins, GA 31099, USA

e-mail: riclheaton@yahoo.com

M. S. Walid $(\square)$

Medical Center of Central Georgia,

840 Pine Street, Suite 880,

Macon, GA 31201, USA

e-mail:mswalid@yahoo.com
}

The presence of endometriosis has a stronger association with typical IC findings on cystoscopy including glomerulations, ulcers, and reduced bladder capacity.

Keywords Interstitial cystitis $\cdot$ Endometriosis . Adenomyosis · PUF PST P ABC

\section{Background}

Chronic or cyclic pelvic pain is a common complaint in women. A large percentage of these cases have been shown to have interstitial cystitis, endometriosis, or both (evil twins) [1-4].

Interstitial cystitis (IC) is thought to be underestimated especially in the younger female population $[5,6]$. It manifests with the triad: pelvic pain, urinary urgency/ frequency, and nocturia. These symptoms may overlap with those of other common gynecologic and urologic conditions and may annoy the patient for years before interstitial cystitis is diagnosed [7]. According to literature, hysterectomy is performed more frequently in patients with undiagnosed interstitial cystitis than in patients with a confirmed IC diagnosis $[2,8]$. Many patients continue to experience persistent pelvic pain despite having had their uterus removed until interstitial cystitis is diagnosed and treated $[2,8]$. Careful history, physical examination, and the use of Pain Urgency and Frequency (PUF) questionnaire and potassium $(\mathrm{KCl})$ sensitivity test (PST) help detect the majority of interstitial cystitis cases. As an alternative to the PST, a symptomatic patient can undergo anesthetic bladder challenge (ABC) [9]. Direct visualization of typical interstitial cystitis lesions in the bladder can be achieved only through cystoscopy with hydrodistension. Laparoscopy is frequently required to exclude other sources of pelvic pain, mainly endometriosis and/or adnexal adhesions $[1,4]$. 
In this paper, we present our experience with cystoscopy and hydrodistension for the diagnosis of IC and put forth a theory explaining the relationship between interstitial cystitis and endometriosis/adenomyosis.

\section{Materials and methods}

This is a retrospective study of 116 patients who have undergone cystoscopy with hydrodistension (distension of the bladder to 70 to $100 \mathrm{~cm}$ of water pressure for $2-5$ minutes). Cystoscopy with hydrodistension was recommended by the National Institute for Diabetes and Digestive and Kidney Diseases (Bethesda, MD, 1987) as an objective test for the diagnosis of interstitial cystitis in research protocols [10]. These patients had cystoscopy with hydrodistension alone for failed office management of presumed interstitial cystitis based on PUF $\geq 10$ with either positive PST, ABC, or both $(\mathrm{N}=10)$ or at the time of certain gynecological procedures, including total laparoscopic hysterectomy $(\mathrm{N}=39)$, total laparoscopic hysterectomy with anterior and/or posterior repair $(\mathrm{N}=3)$, diagnostic or operative laparoscopy $(\mathrm{N}=42)$, anterior and/or posterior colporrhaphy with Prolift placement $(\mathrm{N}=14)$, midurethral sling procedures $(\mathrm{N}=7)$, and myomectomy $(\mathrm{N}=1)$. PUF questionnaires were filled by all patients except three. PST was performed in 68 patients and $\mathrm{ABC}$ in 31 patients. Age range was $15-77$ years and body mass index $17-50 \mathrm{~kg} / \mathrm{m}^{2}$.

We studied the percentage of patients with different cystoscopic findings characteristic of interstitial cystitis, the sensitivity and specificity of the three diagnostic testsPUF, PST, and ABC - regarding interstitial cystitis, and the association between interstitial cystitis and endometriosis/ adenomyosis. Regression analysis and graphing were performed using SPSS v15.

\section{Findings}

According to PUF, 24 patients had a $<10$ score; 72 patients were in the 10-19 range, 13 in the 20-29 range, and four had a score $\geq 30$. Based on the PST result, 45 patients were positive and 23 negative. The $\mathrm{ABC}$ yielded 23 positive and eight negative patients. As per cystoscopic findings, the results were as follows:

1. Sixty-two (53.4\%) patients had typical IC findings with glomerulations and/or Hunner's ulcers and bladder capacity $\leq 800 \mathrm{cc}$.

2. Thirteen (11.2\%) had atypical findings with no glomerulations or less than anticipated, i.e., $<10$ per four separate cystoscopic fields and no Hunner's ulcers; however, bladder capacity was $\leq 800 \mathrm{cc}$.
3. Twenty-five $(21.6 \%)$ had typical glomerulations or Hunner's ulcers, but a bladder capacity $>800 \mathrm{cc}$ or the capacity not documented (only three).

4. Four $(3.4 \%)$ had less than four cystoscopic fields of view with $\geq 10$ glomerulations and bladder capacity $>800$ cc. Some of these patients had positive PST and can be considered as painful bladder syndrome patients of uncertain long-term significance or may just be very early in the course of the disease (IC).

5. Twelve $(10.3 \%)$ had bladder capacity $>800 \mathrm{cc}$ with no or few glomerulations and no Hunner's ulcers.

Pathology reports showed 49 patients had endometriosis. Adenomyosis was diagnosed by pathologists in 22 cases, by ultrasound in two cases, and by hysteroscopy in two cases. Among patients with interstitial cystitis, 60 $(60 \%)$ patients had adenomyosis or endometriosis.

PUF questionnaire had high sensitivity (98\%) with $\geq 5$ score but low specificity ( $0 \%$ ) for IC (first-third groups). The best specificity was found with KCL, 30\% (Fig. 1). Regression analysis showed that $\mathrm{KCl}$ test was the most important predictor of cystoscopic findings $(P<0.05)$. Using these three tests, IC cystoscopic findings (typical, atypical, and no IC) can be predicted in $20 \%$ of cases $\left(R^{2}=.208\right)$.

The fourth and fifth groups of cystoscopic groups were united as the "no IC" group; the third and second as the "atypical IC" group, and the first was called the "typical IC" group. Chi-square analysis showed increasing endometriosis/adenomyosis rate from "no IC" to "typical IC" groups $(P<0.05$; Fig. 2). Also, there were more "typical IC" findings in the endometriosis/adenomyosis group (63.6\% vs. $40 \%, P<0.1$ ) (Fig. 3).

\section{Discussion}

For the last 3 years, our practice has utilized the PUF questionnaire as a screening tool on all annual exams,

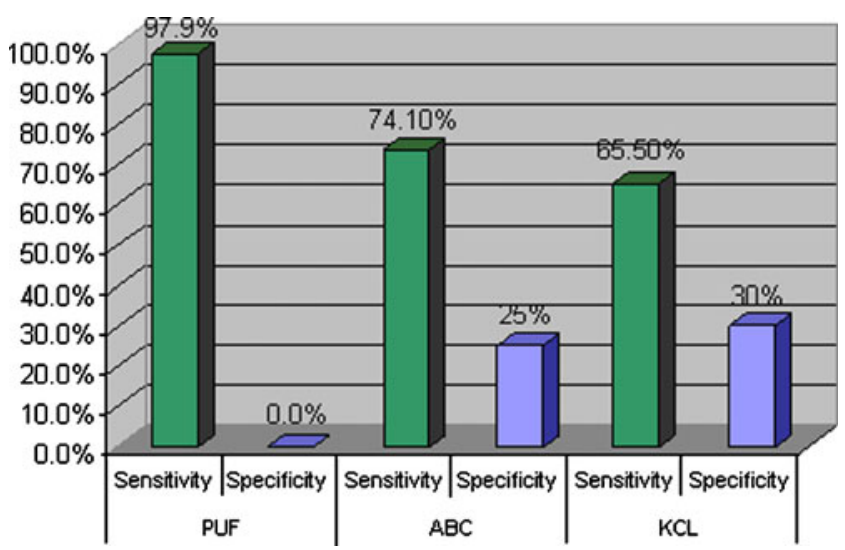

Fig. 1 The sensitivity and specificity of PUF, $\mathrm{KCl}$, and $\mathrm{ABC}$ in regards to IC cystoscopic findings 
Fig. 2 The percentage of concomitant endometriosis/adenomyosis within each cystoscopic category

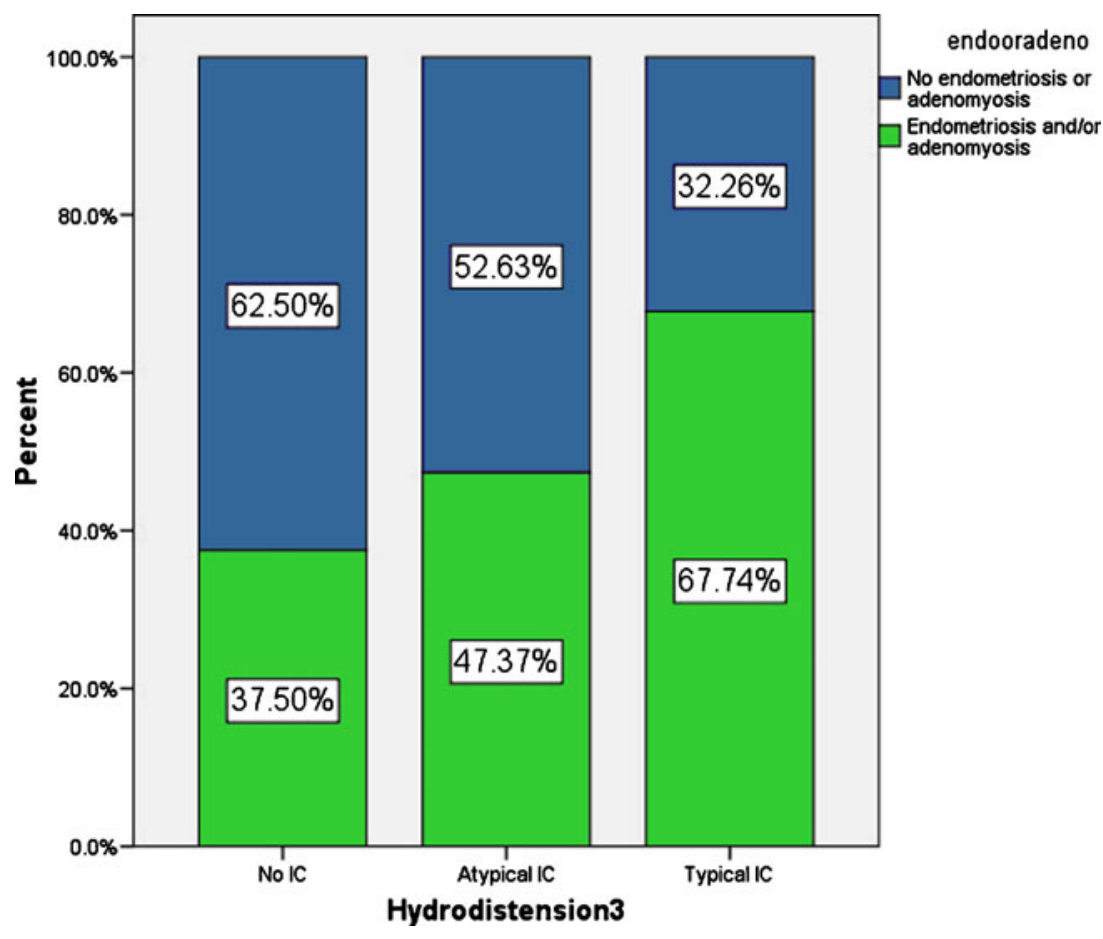

new gynecological patient evaluations, and pelvic pain evaluations. For PUF scores $\geq 10$, PST was recommended. If the patient had indications for gynecological surgery, concomitant cystoscopy with hydrodistension was performed to confirm the diagnosis, predict response to therapy based on bladder findings, and to induce temporary remission enough to give medical therapy time to work.

Fig. 3 The percentage of concomitant typical IC within each endometriosis/adenomyosis group

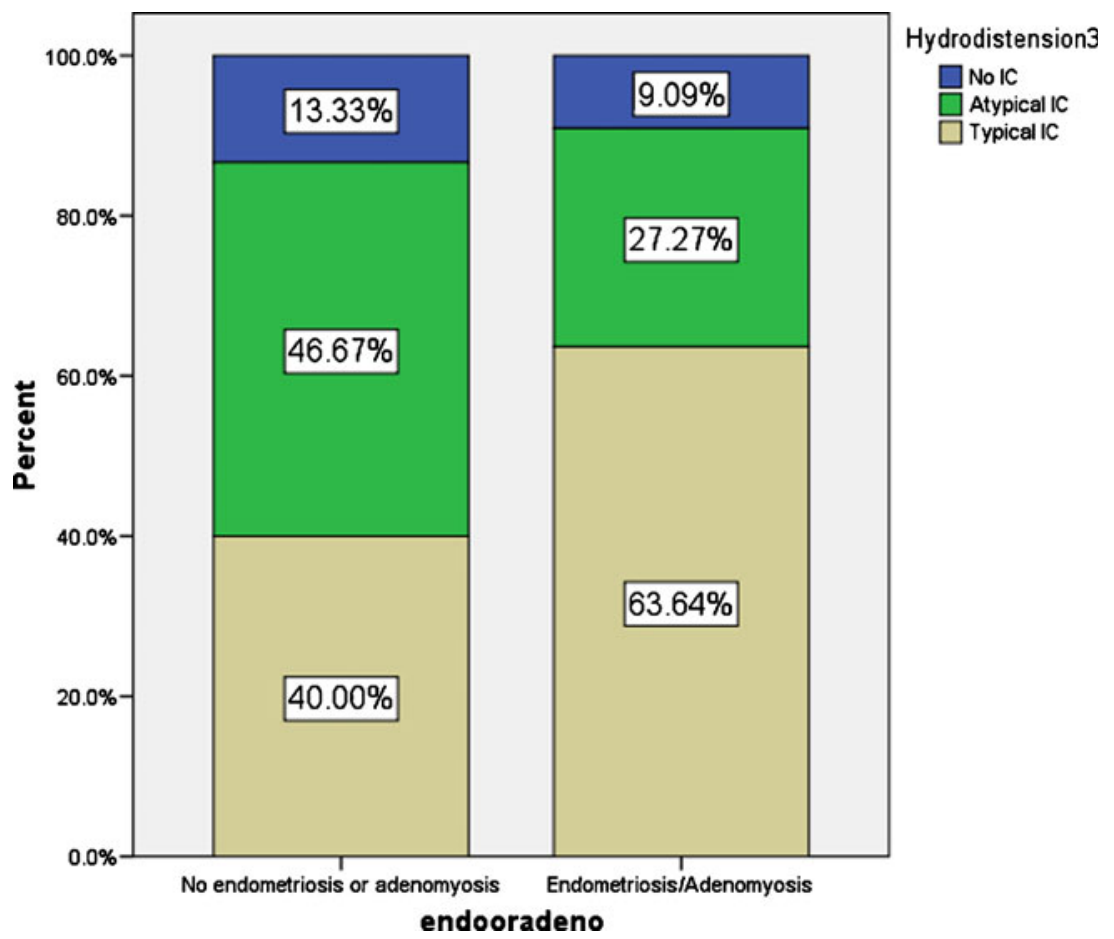

dooradeno
Our study is a retrospective case-series analysis based on known implemented criteria of routine PUF questionnaire screening with intent to do PST, $\mathrm{ABC}$, and/or cystoscopy with hydrodistension in patients that needed gynecological surgery who were felt to have interstitial cystitis as suggested by an elevated PUF score, a tender trigone on physical exam, or complaints of nocturia, a strong marker for interstitial cystitis [11]. Hence, with a 
known history of endometriosis or adenomyosis (intrauterine endometriosis), we erred on the side of further evaluation with cystoscopy and hydrodistension in patients with these findings as well.

The majority of these patients were undergoing other concomitant GYN procedures which made them available for confirmation of diagnosis and placement into diagnostic groups. Our observations allow us to postulate a strong primary correlation between interstitial cystitis and endometriosis/adenomyosis. These data were obtained from cystoscopy with hydrodistension, pathology, hysteroscopy, and preoperative ultrasound imaging reports. They are consistent with findings from other studies showing increasing incidence of coexistence of interstitial cystitis and endometriosis/adenomyosis [1, 4, 5]. We found a $60 \%$ association rate between interstitial cystitis and endometriosis/adenomyosis, but it is probably higher given the inability to diagnose endometriosis or adenomyosis in some types of procedures (e.g., Prolift procedure) or due to unavailiability of pathology reports from prior hysterectomy in some patients. Our study expands on previous reports by showing that the presence of endometriosis has a stronger association with typical IC findings on cystoscopy, including glomerulations, ulcers, and reduced bladder capacity.

The antiproliferative factor (APF) and the heparinbinding epidermal growth factor-like growth factor (HBEPF) identified in bladders affected by interstitial cystitis seem to play a role in the relationship between interstitial cystitis and endometriosis/adenomyosis [12, 13]. Interstitial cystitis seems to occur as a result of misbalance in the concentrations of APF and HB-EPF with resultant inhibition of bladder epithelial repair $[14,15]$. This would also explain the 8:1 incidence of interstitial cystitis in women compared with men $[7,16]$. The fact that men may also develop interstitial cystitis suggests that other stillunknown initiators may shift the balance of APF and HB-EPF. The effect of the antipoliferative factor would also explain many of the symptoms associated with endometriosis like menorrhagia, metrorrhagia, and infertility. Similarly, many of the symptomatic bowel problems associated with endometriosis like diarrhea, bloating, and cyclic rectal bleeding (even when no colorectal endometriosis is found) could also be explained by the effect of the antipoliferative factor $[12,17]$. This is further supported by the exacerbation of symptoms of interstitial cystitis in late luteal and menstrual phases when the inflammatory response to endometriosis/adenomyosis is most intense. This concept along with genetic stability of the AFP-HB-EPF homeostasis can explain the puzzling finding that some women with minimal endometriosis have severe IC symptoms while others with severe endometriosis have minimal or no IC symptoms.
One would theorize that, in a large number of patients who go into remission after hydrodistension, a positive balance in favor of endothelial proliferation factors occurs promoted by tissue injury at the time of hydrodistension overcoming the effect of endothelial antiproliferation factors. This way of viewing the pathologic process might also explain the effect of pentosan polysulfate sodium in putting patients into remission.

We believe the categorization of cystoscopic findings as set forth above is essential for prognosis of interstitial cystitis patients and assists in encouraging those with reduced bladder capacity to adhere to their dietary restrictions and medications while allowing less aggressive treatment of those with preserved bladder capacity who may represent women who have a less fulminant course that may be candidates for intermittent therapy.

In the USA, cystoscopy with hydrodistension is still considered optional in the evaluation of patients with symptoms suggestive of interstitial cystitis, including urinary urgency, frequency, nocturia, and/or pelvic pain [18, 19]. However, cystoscopy with hydrodistension is the only objective method allowing direct visualization of bladder mucosal abnormalities and thus has a diagnostic and a prognostic value [18]. Cystoscopy with hydrodistension is the only objective means that allows direct visualization of bladder mucosal abnormalities and thus has a diagnostic and a prognostic value. It thus helps diagnose and categorize patients into those with a classic picture of IC including mucosal abnormalities and those without [18]. Unfortunately, the diagnosis of IC is usually delayed because of lack of enthusiasm about cystoscopy with hydrodistension [7, 20]. A recent European study showed correlations between cystoscopic findings, maximal bladder capacity, and bladder histology and considered cystoscopy with hydrodistension and biopsy mandatory for further typing of patients with bladder painful syndrome [21]. The questionnaire and supplementary tests may suffice if the patient responds to medical therapy and has exacerbations to known dietary indiscretions, in other words, running a typical course and responding to therapy. However, failing to proceed to performing cystoscopy and hydrodistension in nonresponding patients or those with risk factors for bladder cancer would be irresponsible $[18,20]$. If we follow the logic of those that want to deemphasize the use of cystoscopy with hydrodistension to other organ systems, then why do we perform endoscopy in patients with persistent gastrointestinal complaints?

In addition, cystoscopy with hydrodistension has a therapeutic effect with more than half the patients reporting improvement in symptoms for an average of 2 months $[20,22]$. This, by itself, is a very helpful diagnostic marker of interstitial cystitis. More than half of patients report improvement in symptoms after this procedure with a mean duration of 2 months [20]. 
Gynecologists should be trained in cystoscopy with hydrodistension and in the care and treatment of women with interstitial cystitis and the commonly associated syndrome of myofascial pelvic pain and spasm. We need to take the lead in dealing with this very debilitating and now understood to be fairly common condition (as many as $12.6 \%$ of the female population [23]) where delayed diagnosis continues to be habitual leading to untold and unnecessary human suffering.

\section{References}

1. Paulson JD, Delgado M (2007) The relationship between interstitial cystitis and endometriosis in patients with chronic pelvic pain. JSLS 11(2):175-181

2. Butrick CW (2007) Patients with chronic pelvic pain: endometriosis or interstitial cystitis/painful bladder syndrome? JSLS 11(2):182-189

3. Chung MK et al (2002) The evil twins of chronic pelvic pain syndrome: endometriosis and interstitial cystitis. JSLS 6(4):311-314

4. Chung MK, Chung RP, Gordon D (2005) Interstitial cystitis and endometriosis in patients with chronic pelvic pain: The "evil twins" syndrome. JSLS 9(1):25-29

5. Rackow BW et al (2009) Interstitial cystitis is an etiology of chronic pelvic pain in young women. J Pediatr Adolesc Gynecol 22(3):181-185

6. Parsons CL, Tatsis V (2004) Prevalence of interstitial cystitis in young women. Urology 64(5):866-870

7. Mouracade $P$ et al (2008) Using the intersitital cystitis new diagnostic criteria in daily practice: about 156 patients. Prog Urol 18(10):674-677

8. Chung MK, Jarnagin B (2009) Early identification of interstitial cystitis may avoid unnecessary hysterectomy. JSLS 13 (3):350-357

9. Evans RJ, Sant GR (2007) Current diagnosis of interstitial cystitis: an evolving paradigm. Urology 69(4 Suppl):64-72

10. Gillenwater JY, Wein AJ (1988) Summary of the national institute of arthritis, diabetes, digestive and kidney diseases workshop on interstitial cystitis, national institutes of health, Bethesda, Maryland, August 28-29, 1987. J Urol 140(1):203-206

11. Richter B, Hesse U, Hansen AB et al (2010) Bladder pain syndrome/interstitial cystitis in a Danish population: a study using the 2008 criteria of the European Society for the Study of Interstitial Cystitis. BJU Int 105:660-667

12. Keay $S$ et al (2004) Antiproliferative factor, heparin-binding epidermal growth factor-like growth factor, and epidermal growth factor in men with interstitial cystitis versus chronic pelvic pain syndrome. Urology 63(1):22-26

13. Keay S et al (2000) Bladder epithelial cells from patients with interstitial cystitis produce an inhibitor of heparin-binding epidermal growth factor-like growth factor production. J Urol 164(6):2112-2118

14. Keay S, Warren JW (1998) A hypothesis for the etiology of interstitial cystitis based upon inhibition of bladder epithelial repair. Med Hypotheses 51(1):79-83

15. Chai TC, Keay S (2004) New theories in interstitial cystitis. Nat Clin Pract Urol 1(2):85-89

16. Mouracade P, Saussine C (2008) Interstitial cystitis in 2008. Prog Urol 18(7):418-425

17. Kim J, Keay SK, Freeman MR (2009) Heparin-binding epidermal growth factor-like growth factor functionally antagonizes interstitial cystitis antiproliferative factor via mitogen-activated protein kinase pathway activation. BJU Int 103(4):541-546

18. Carr LK et al (2009) Diagnosis of interstitial cystitis June 2007. Can Urol Assoc J 3(1):81-86

19. Rabe HH, Gotoh M, Momose H (2003) The place of cystoscopy and hydrodistension in the diagnosis of interstitial cystitis: a potpourri of opinions emanating from an International Consultation on IC in Kyoto, Japan. Int J Urol 10(Suppl):S16-S18

20. Ottem DP, Teichman JM (2005) What is the value of cystoscopy with hydrodistension for interstitial cystitis? Urology 66(3):494-499

21. Wyndaele JJ, Van Dyck J, Toussaint N (2009) Cystoscopy and bladder biopsies in patients with bladder pain syndrome carried out following ESSIC guidelines. Scand J Urol Nephrol 43(6):471-475

22. Erickson DR, Davies MF (1998) Interstitial cystitis. Int Urogynecol J Pelvic Floor Dysfunct 9(3):174-183

23. Rosenberg MT, Hazzard M (2005) Prevalence of interstitial cystitis symptoms in women: a population based study in the primary care office. J Urol 174(6):2231-2234 